\title{
Robot-Based Fiber Spray Process for Small Batch Production
}

\author{
Edgar Schmidt ${ }^{1}$, Jonas Winkelbauer ${ }^{2}$, Georg Puchas ${ }^{2}$, Dominik Henrich ${ }^{1}$, and \\ Walter Krenkel ${ }^{2}$ \\ 1 University of Bayreuth, Chair for Robotics and Embedded Systems, \\ D-95440 Bayreuth, Germany, \\ edgar.schmidt@uni-bayreuth.de \\ ${ }^{2}$ University of Bayreuth, Department for Ceramic Materials Engineering, \\ D-95440 Bayreuth, Germany, \\ jonas.winkelbauer@uni-bayreuth.de
}

\begin{abstract}
Fiber spraying processes are used in the production of fiberreinforced composites, but are performed mainly manual. Given various possibilities for automating such processes, only a few are suitable for the production in small and medium-sized enterprises. We present an approach on robot-based automation of fiber spraying processes. This approach is characterized by its easy programmability of the robot system using the playback programming paradigm. We simulated the reachability and accessibility of our robot-based automation approach and present our prototype system, which can be used for the production of small batches of fiber-reinforced composites.
\end{abstract}

Keywords: industrial robotics, automation technologies, flexible manufacturing, small batch production, intuitive robot programming, playback programming, fiber spraying

\section{Introduction}

Fiber spraying processes (FSPs, also called spray lay-up processes) are one of the most commonly used production methods for short fiber-reinforced composites [1]. While often used in the production of glass and carbon fiber composites, FSPs are not commonly used for ceramic matrix composites, such as oxide fiber composites (OFC). However, recent research shows that applying an FSP in context of manufacturing of OFC can save over one third of the production costs [2]. A further reduction of the production costs and an increase of the throughput is possible with the automation of such processes.

Robot-based automation is still only rarely spread in small and medium-sized enterprises (SMEs), even though a low cost and high quality production is possible with their use [3]. The slight use of robot-based automation is largely due to the production of small batches, which is characterized by different phases of robot use with frequent reconfiguration and changeover of the robot systems [4]. 


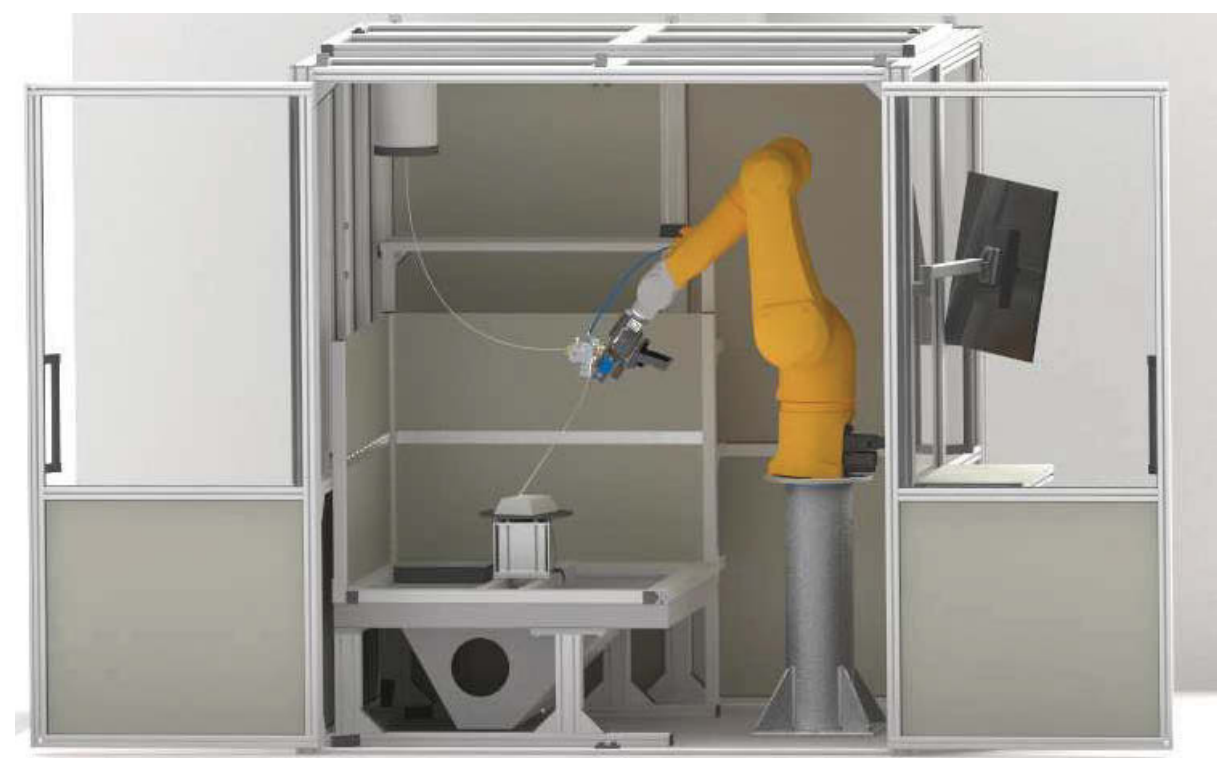

Fig. 1. Proposed cell for robot-based fiber spraying process.

A fast reconfiguration can be achieved through a speed up of the robot programming, which is possible when using intuitive robot programming approaches like playback programming [5][6][7]. With the use of such techniques a cost-efficient production of small batches could be possible.

We envision a robot-based automation approach of FSPs, with the aim to perform various FSP with different fiber materials in one cell with a shorter changeover time. The resulting system should be quickly and spontaneously programmed by non-experts in robot programming, which are experts in the given process, who we call domain experts.

In this paper, we present a novel robot-based production system for the production of OFC, in which a robot is covering a positive or negative mould with a fiber slurry mixture (Figure 1). The system can be extended to other kinds of FSP like glass fiber spraying. The robot in the cell is further designed to be programmed with the intuitive playback programming paradigm. Section 2 gives an brief overview of the related work regarding the automation of FSPs and their programming frameworks. Then, we explain in Section 3 our robotbased automation approach in detail. The simulation and development of our prototypical system is described in Section 4. In Section 5, we summarize and conclude the paper.

\section{Related Work}

Most of the commercially available fiber spraying systems are manual operated systems, on which the various required components are installed, so that they can 
be used like a paint spray gun [8][9]. When used, these manually operated systems expose the domain expert to polluted air (overspray, fibers) during the spraying. Also, the quality of the produced composite depends heavily on the expertise of the operator with every workpiece. So, the quality of produced composites is hardly reproducible and the error rate is high. An automation approach should put a stop to these disadvantages.

To the best of our knowledge, there is only one prototypical system for robotbased FSPs using off-line programming, which can be characterized by graphical programming and virtual reality [10]. Different advantages of this approach are given. One of these is, that during the production no worker is exposed to the polluted air. Also, the use of a rotary table as an external rotation axis enables the processing of complex shapes. Further, layers with uniform thickness can be applied repeatedly. However, the off-line programming approach is only of limited suitability for small batch production since such programming framework requires a special training in robot programming. A new approach should put a focus on an intuitive robot programming framework, which exploits the process specific knowledge of the operator direct.

Using on-line programming paradigms could allow a cost-efficient production, for example by hand guiding of the robot tool head, also called kinesthetic guiding [6][11]. One of the approaches using kinesthetic guiding is playback programming and has the advantage, that process-specific knowledge of a domain expert continues to be incorporated directly into the programming process of the robot, as already shown for other processes in [7][11][12][13]. A new approach to robot-based automation of FSP for small batch production can also take advantages from this programming method. For this reason, a novel approach on robot-based automation of FSP should integrate a playback programming framework.

\section{Automation of Fiber Spraying}

In the following, we describe our approach in automation of FSPs. First, the process itself is explained. Second, we consider important design criteria for an automation approach. Then, we explain our system design in detail. Finally, we describe how the robot programming framework of the process is designed.

\subsection{Fiber Spraying Process for OFC}

In an FSP for the manufacturing of OFCs aplications, continuous oxidic fiber bundles (rovings) are chopped to uniform length by a cutting unit immediately before being ejected out of the cutting unit and into a slurry spray, by which they are entrained. Due to the angle and the distance between the tool head (cutting unit and spray gun) and the mould surface the bundles are infiltrated during flight with the slurry, as shown in Figure 2. The infiltrated fiber bundles, with slurry being inside the fiber bundles as well as between the fiber bundles reach the mould surface and are orientated randomly. 


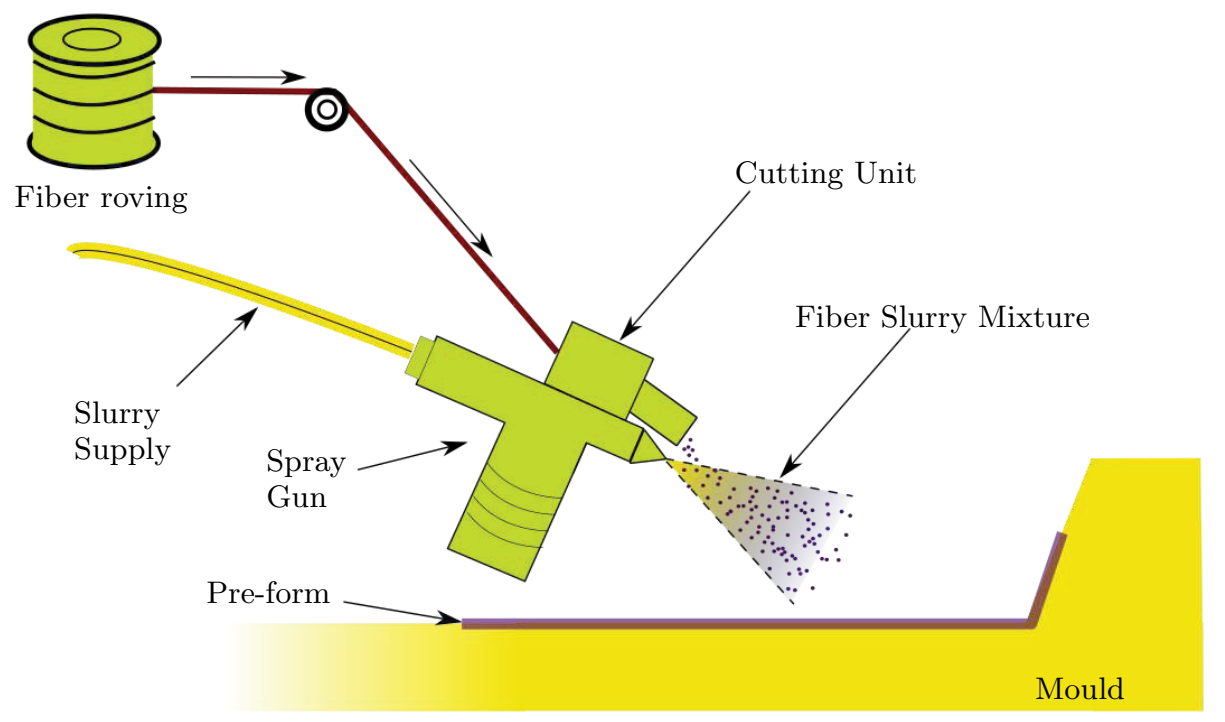

Fig. 2. Schematic representation of a manual fiber spraying process.

If desired, the thus prepared pre-impregnated fiber preform (prepreg) can be conditioned in a climate chamber to adjust a defined water content, stacked and laminated. In case of flat prepregs, they are stacked to a laminate and laminated or directly compressed under pressure, if they are not already corresponding to the final form. After consolidation in a drying chamber, the green bodies are sintered for two hours at $1225^{\circ} \mathrm{C}$. The short fiber bundle reinforcement allows more complex shapes than it is the case with fabrics, without fear of damaging the fiber bundles or getting delaminations due stresses in the fabrics and a resulting resilience.

As already mentioned this method can be used on the one hand as a primary shaping process, such as by spraying on a positive or a negative mold, or as a shaping process using the flat prepreg to shape more complex geometries, which are not accessible using fabric-based prepregs. On the other hand the spraying process build flat prepregs which can be processed like conventional prepregs. The big advantage here is the increased drapability of the short fiber prepregs compared to the fabric prepregs. Furthermore, the random arrangement of the fibres results in area-isotropic mechanical properties.

\subsection{Evaluation and Design Criteria}

The design of an automation approach for an FSP depends on various criteria. A very important criterion is the possibility to adjust different parameters of the process. One of these parameters is the image of the spay pattern from which the 
fibers are captured. This spray pattern defines the slurry spray which infiltrates the fiber bundles, entrains them towards the mould and so defines also the resulting material properties of the workpiece. An automation approach must be able to adjust the slurry spray regarding flow volume and spray pattern, according to the objectives of a workpiece. In addition to the slurry spray, the fiber flow needs also to be adjustable so that material properties can be modified. This plurality of free variables allows the production of a wide variety of workpieces with different material properties.

In addition, economic criteria have to be considered, which are directly related to the production costs. One of these criteria is the overall material consumption of fiber and slurry. Minimizing the material consumption, e.g. the amount of overspray which does not form the workpiece, will lead directly to lower production costs when using high-expensive fibers. Another criterion is the production time, whose minimization will increase the throughput of produced components. A further time related criterion is the setup time for the production of a single component. This time is required for the configuration of the system, when a new component of the same material class has to be produced. Reducing the configuration time is essential for the production of small batches as described in Section 1. Only if all of the listed criteria are minimized to its best, a cost-efficient production of small batches can be reached.

Finally, process-specific criteria must be considered. The first two criteria are to keep a fixed orientation and distance from the tool head to the mould and to guarantee the accessibility of the spraying on the mould. This results in an optimal application of the fiber slurry mixture. The accessibility is challenging due to the fact that we want to accomplish a complete coating even for moulds with complex geometries. The last criterion is the ability to generate a homogeneous coverage over the complete mould. Achieving a homogeneous coverage leads to uniformly distributed material properties (e.g. the tensile or bending strength) over the entire geometry, which can be used as an quality measure for a correctly produced workpiece.

\subsection{Robot-based Automation}

As automation approach, we propose a robot system, which manipulates standard components of manually operated systems for FSPs, focusing on the production of small components with a maximum size of up to $500 \times 500 \times 500 \mathrm{~mm}^{3}$. First, the structure of the cell is explained, then the concept of the tool head.

The boundaries of the cell are formed by a cabin of system profiles (W: $2000 \mathrm{~mm} \times \mathrm{H}: 2200 \mathrm{~mm} \times \mathrm{D}: 1800 \mathrm{~mm}$ ). The cabin is sealed by windows and resealable doors made of acrylic glass, so that overspray and fibers are contained within the cell and the production can be monitored from outside. In addition, all control elements are mounted outside the cell so that the presence of a person inside the cell is not necessary while the FSP is running. Within the cell, an underfloor downdraft table $(\mathrm{W}: 1080 \mathrm{~mm} \times \mathrm{H}: 460 \mathrm{~mm} \times \mathrm{D}: 1195 \mathrm{~mm})$ is set up, where a mould can be positioned. In the middle of the downdraft table a programmable turntable is installed allowing the rotation of the mould through 

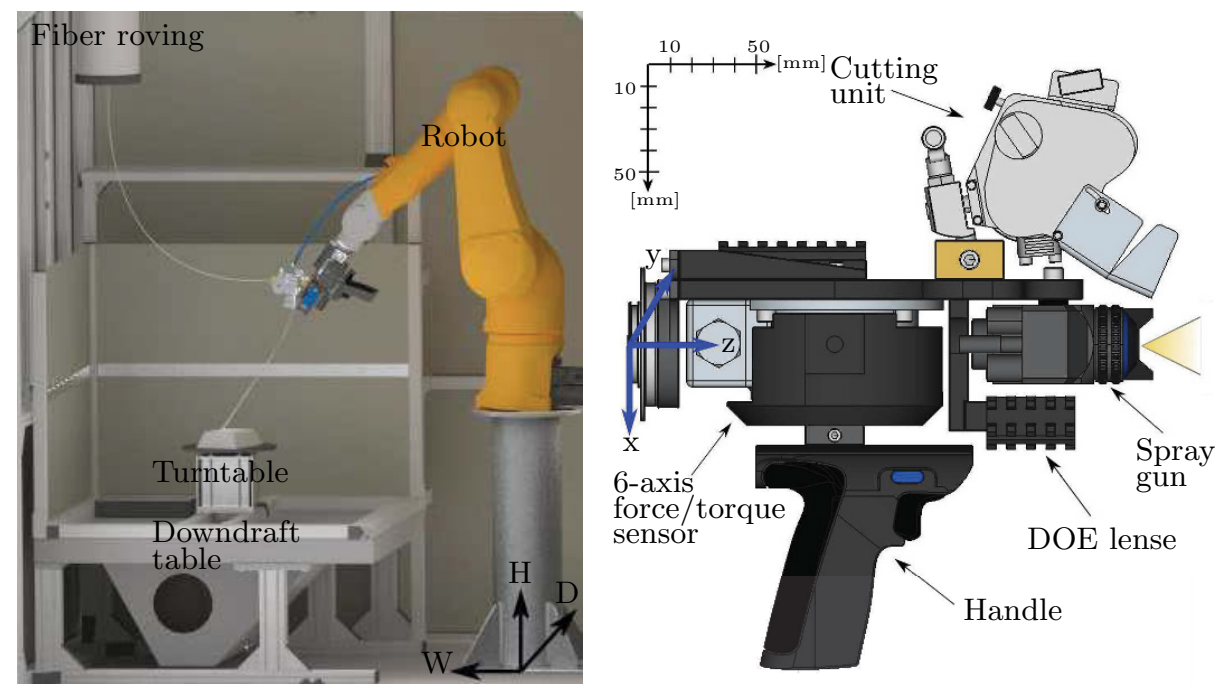

Fig. 3. Proposed concept of a robot-based fiber spraying cell. Left: Interior of the fiber spraying cell. Right: Tool head design with possibility of kinesthetic guiding.

this external axis. Additional degrees of freedom can be added by extending the turntable to a two-axis turntable. The downdraft table limits the intended working space of a six degree of freedom robot, which is mounted in front of the downdraft table (Figure 3, left).

The possibility of programming by kinesthetic guiding was taken into consideration in the design of the tool head (Figure 3, right). A force/torque sensor is mounted in z-direction from the flange. A handle is additionally attached on the force/torque sensor, through which the guiding of the robot arm is enabled. The automatic spray gun is installed in front of the tool head. The slurry flow volume as well as the spray pattern are adjusted by a programmable system of proportional pressure regulators. The fibers are fed via a cutter mounted over the spray gun. The fiber roving supply is mounted on the ceiling of the cell. The amount of fiber throughput is also controlled by the proportional pressure regulators. A diffractive optical element projects a pattern onto the mould allowing the estimation of the spray cone and thus the application of material without a material flow. The resulting tool head has a weight of $4 \mathrm{~kg}$, which can also be mounted on lightweight robots.

Complying a fixed orientation and distance to the mould and achieving homogeneous layer thicknesses is possible due to the programmability and repeatability of robot movements. At the same time an increase in the throughput of produced ceramics can be achieved by the autonomous execution of robot programs. The accessibility of the mould is guaranteed by the use of a turntable, which also reduces the robot wear. 


\subsection{Robot Programming}

An approach in robot-based automation for small batches requires a programming technique, which can quickly provide robot programs for covering various moulds fast. Textually created robot programs require the specialized knowledge of a robotics expert. Further, a new robot program for each workpiece or groups of workpieces in case of simple geometries (plates, tubes) is needed. For this reason, the robot has been equipped with the ability to be guided kinesthetically by a domain expert. The kinesthetically guiding allows the use of the playback programming paradigm [6]. Given sensor data from a force/torque sensor, the robot moves in answer to this input of the operator, who can be a domain expert. While the robot arm is guided by the operator through the spraying task, the system records robot configurations in a fixed cycle and generates a robot program with the demonstrated trajectory. After this programming stage, the robot program can be played back so that he can execute the intended task.

The use of the playback programming paradigm allows the quick teaching of trajectories for geometrically complex moulds, which would be time and cost consuming by a textual programming approach. Also, kinesthetic programming is intuitive and can be done by domain experts. Further, our system allows, that the playback programming can be done without material flow and is so resourcesaving. The resulting program can be used for the automatic production while the domain expert can address oneself to other tasks. Finally the reproducibility of robot movements guarantees that a fault-free program can be executed again and thus reduces the error rate of the production.

\section{Prototypical System Development}

In this section, we describe the development of our prototypical system. First, we describe the analysis of our approach through simulation. Then, we show how our first prototypical system was designed and give an overview about its use.

\subsection{Simulation}

Given geometrical data of the cell and tool head in form of CAD-models, we simulate the reachability of the robot for different poses. Our aim is to check, whether the optimal distance for covering a mould is achievable. The optimal distance is given by our first application, the process given in [2]. With the optimal distance, we generate distributed poses on a hemisphere around the position where the mould will be placed. After that, we check if the robot kinematic can reach the poses on the hemisphere. Additionally, we let the simulated robot move into the reachable positions, using a collision check between the robot and the downdraft table to guarantee the accessibility of the poses.

For simulation we use the Stäubli Robotic Suite [14] (Figure 4, left). We sample 500 poses and a distance from spray jet to mould of $400 \mathrm{~mm}$ is used, resulting in a overall distance from flange to the hemisphere center point of 645 

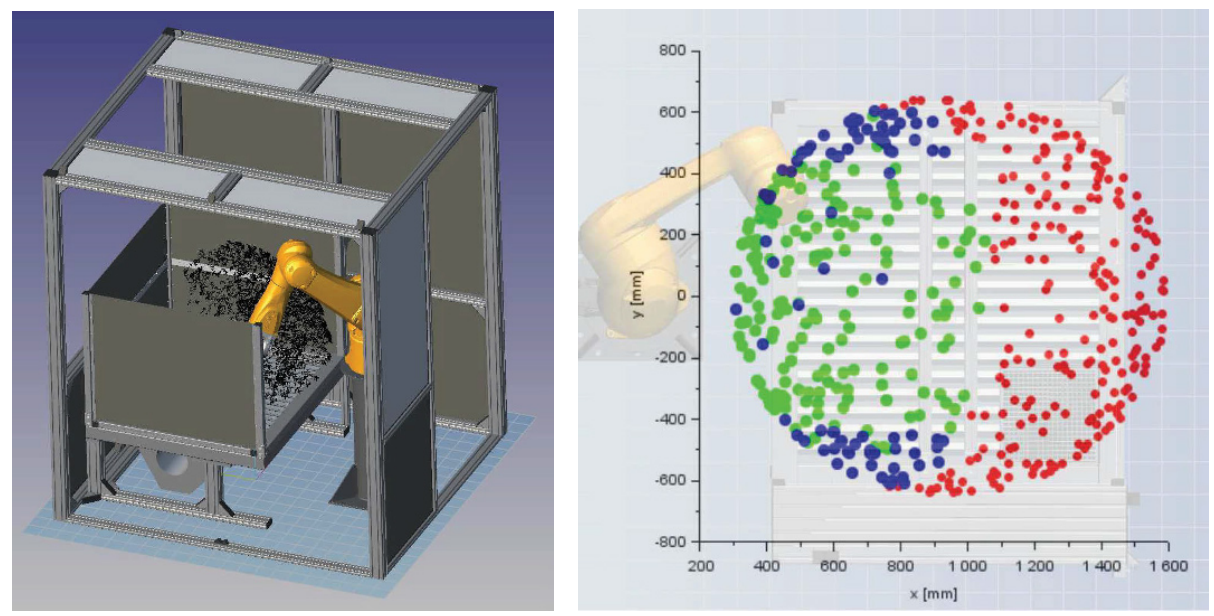

Fig. 4. Simulation of our approach on robot-based fiber spraying process. Left: Simulation setup with reachable poses marked as black dots. Right: Detailed results of our simulation with classification into not reachable poses (red), reachable poses but the movement causes a collision (blue) and collision-free reachable poses (green).

mm. 263 of the generated poses were reachable, in which 85 result in collisions with the robot cell while moving. So, the remaining 178 poses are accessible from the robot without collision. Eight collision-related poses are characterized by being in an area, which is dominated by accessible poses. The results are shown in Figure 4, right.

One of our conclusions from the simulation is, that only with the use of a turntable geometrical high complex moulds with the required maximum size can be covered complete. Another conclusion is, that for plain geometries, the reachability and accessibility for the optimal distance can be guaranteed with our concrete approach. Our approach, which uses a combination of turntable and robot, is thus sufficient for the required process and workpieces.

\subsection{Prototypical System}

For our first prototypical system we use a Stäubli TX2-90L [15] as robot and an ISEL IT 116 [16] controller for the turntable. The control of the different pressures needed for the material feed is given by a system of proportional pressure regulators. Slurry output is enabled through a spray gun, which is a commercially available system suited for the use mounted on a robot. The turntable and pressure regulators can also be controlled by the manual robot control pendant. Five pressures can be configured via the regulators: two defining the slurry, one for setting the actual slurry output to ON/OFF, one for controlling the volume flow of the slurry, and the last for the rotation speed of the chopper unit. A force/torque sensor provides the input data for the playback programming. All components are connected and controlled via the controller of the robot. 


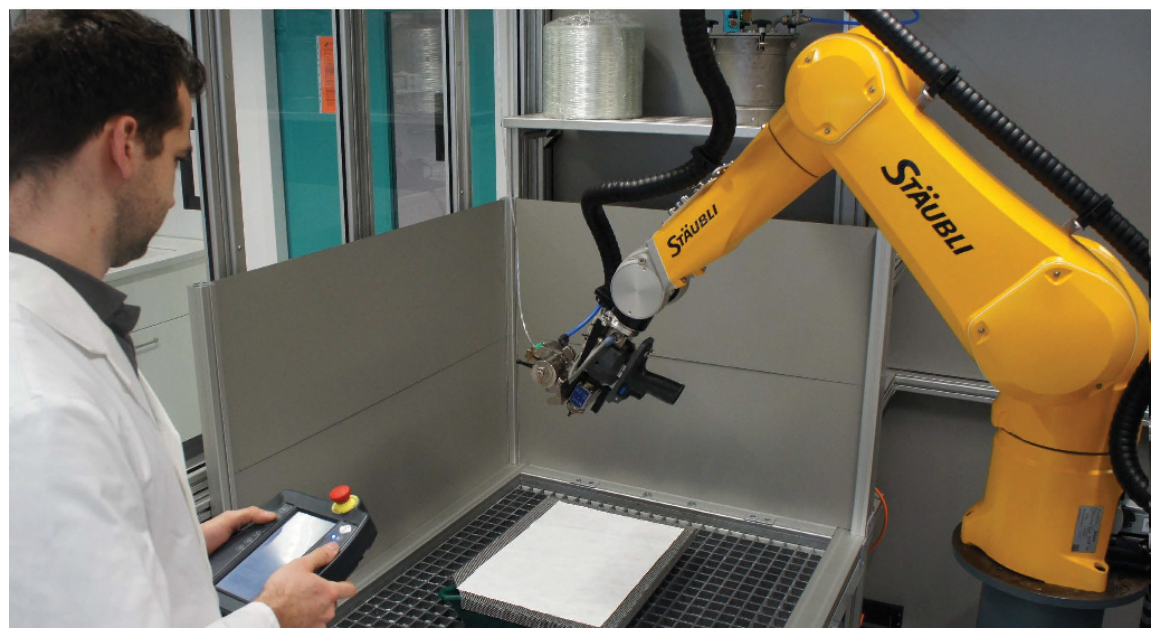

Fig. 5. An example use of our prototypical system for robot-based fiber spraying process. The operator utilizes the manual control pendant for programming.

Given a mould to cover, the operator teaches the required trajectory for the robot by kinesthetic guiding. The playback programing without material feed is defined through the user interface of the manual pendant control before the programming stage begins. Also, the turntable and the pressure regulators are configurable with the manual pendant control, as shown in Figure 5. After teaching, the trajectory can be stored and reloaded for a later production. In the execution stage, the trajectory is replayed with temporal synchronization between the robot, the regulators and the turntable.

This prototypical system allows the playback programming of geometrical complex trajectories with a one shot demonstration, resulting in the fast production of small batches of new workpieces. The quality of the programmed trajectory depends on the skill of the system operator and is suitable for workers with expertise in the given process, but can easily used by them without special training in robot programming.

\section{Conclusion and Future Work}

In this paper, a novel robot-based automation approach for production of fiber composites with FSPs is proposed. The developed prototypical system can be used for the production of OFCs. Reachability and accessibility is guaranteed through simulation. The presented playback programming framework allows the programming of the robot in a new way by a domain expert without special training in robot programming. However, the presented playback programming framework is still not sufficient for a small batch production of geometrical high complex workpieces. For this reason, we will use in future work a playback programming framework which allows the intuitive programming of such tasks using 
video editing concepts [5]. Also, this approach will be extended with functionalities for supporting the domain expert, like the simulation of the process or the optimization of the programmed trajectory before execution.

\section{Acknowledgements}

This work has partly been supported by the European Regional Development Fund (ERDF) under the project "Roadmap zur flexiblen Produktion individueller Produkte" (Roadmap flexPro).

\section{References}

1. Witten, E., Mathes, W., Sauer, M., Kühnel, M.: Composites-Marktbericht 2018: Marktentwicklungen, Trends, Ausblicke und Herausforderungen. Carbon Composites eV und Industrievereinigung Verstärkte Kunststoffe eV, 2018.

2. Puchas, G., Krenkel, W.: Neuartige Herstellungsverfahren für oxidkeramische Faserverbundwerkstoffe, DGM-dIALOG 2018, pp. 34-41, 2018.

3. Perzylo, A., et. al.: SMErobotics Smart Robots for Flexible Manufacturing. IEEE Robotics and Automation Magazine, pp. 78-90, 2019.

4. Dietz, T., Schneider, U., Barho, M., Oberer-Treitz, S., Drust, M., Hollmann, R., Hägele, M. : Programming System for Efficient Use of Industrial Robots for Deburring in SME Environments, ROBOTIK 2012, VDE, pp. 1-6, 2012.

5. Riedl, M., Henrich, D.: A Fast Robot Playback Programming System Using Video Editing Concepts. Tagungsband des 4. Kongresses Montage Handhabung Industrieroboter, Springer Vieweg, pp. 259-268, 2019.

6. Lozano-Pérez, T.: Robot Programming, Proceedings of the IEEE, pp. 821-841, 1983.

7. Ang Jr. M. H., Lin W., Lim S. Y.: A walk-through programmed robot for welding in shipyards. Industrial Robot: An International Journal, pp. 377-388, 1999.

8. Faserspritzanlagen Wolfangel. http://wolfangel.com/faserspritzanlagen/

9. FPR Faserspritzanlage Pultex. https://www.pultex.de/frp-faserspritzanlage/

10. Aßhoff C.: Herstellung von Tailored Composites, Fraunhofer WKI, 2016. https://www.wki.fraunhofer.de/content/dam/wki/de/documents/Mediathek/ themen/hofzet/HOFZET_Faserspritzen_2016-10.pdf

11. Schraft, R. D., Meyer, C.: The need for an intuitive teaching method for small and medium enterprises. VDI BERICHTE, pp.95-104, 2006.

12. Meyer, C., Schraft, R. D.: An intuitive teaching method for small and medium enterprises. Intelligent Production Machines and Systems, Elsevier Science Ltd., pp. 568-571, 2006.

13. Meyer, C., Hollmann, R., Parlitz, C., Hägele, M.: Programming by Demonstration for Assistive Systems - Intuitive Programming of Welding and Gluing Trajectories. it-Information Technology, pp. 238-246, 2007.

14. Stäubli Robotics Suite. https://www.staubli.com/en-de/robotics/product-range/ robot-software/pc-robot-programming-srs/

15. TX2-90 industrieller Roboterarm Stäubli. https://www.staubli.com/de-de/ robotics/produktprogramm/roboterarme/6-achs-roboter/tx2-90/

16. IT 116 Flash single axis controller isel Germany AG. https://www.isel.com/en/ single-axis-controller-it-116.html

17. ATI Industrial Automation: F/T Sensor Gamma IP65. https://www.ati-ia.com/ products $/ \mathrm{ft} / \mathrm{ft} \_$models.aspx?id=Gamma+IP65 
Open Access This chapter is licensed under the terms of the Creative Commons Attribution 4.0 International License (http://creativecommons.org/licenses/by/4.0/), which permits use, sharing, adaptation, distribution and reproduction in any medium or format, as long as you give appropriate credit to the original author(s) and the source, provide a link to the Creative Commons license and indicate if changes were made.

The images or other third party material in this chapter are included in the chapter's Creative Commons license, unless indicated otherwise in a credit line to the material. If material is not included in the chapter's Creative Commons license and your intended use is not permitted by statutory regulation or exceeds the permitted use, you will need to obtain permission directly from the copyright holder. 